control media alone or with $1 \mathrm{nM}$ ATXII in presence/absence of either $10 \hat{\mathrm{I}}^{1 / 4} \mathrm{M}$ ranolazine (Ran; $\mathrm{Na}_{\mathrm{P}}$ blocker) or $5 \mu \mathrm{M}$ BAPTA-AM. All data were expressed as mean \pm SEM. Differences among groups were tested by ANOVA followed by Bonferroni post-hoccomparison. The null hypothesis was rejected at $\mathrm{p}<0.05$.

Under control conditions, HL-1-6 cells and MEFs exhibited 24 hours Bmal1 circadian rhythms with a period of 26.09 \pm 0.77 hours $(n=8)$ and $25.22 \pm 0.26$ hours $(n=8)$, respectively. The Bmal1 rhythmicity was highly disrupted in HL-1-6 cells only when incubated with ATXII, with a 10 hours shortening $(17.06 \pm 1.07$ hours; $n=8 ; p<0.05)$ in their period. The effect of ATXII on HL1-6 cells was reversed in presence of Ran (25.32 $\pm 0.97 ; \mathrm{n}=8 ; \mathrm{p}<0.05$ vs ATXII). In fura2-AM loaded cells, $\left[\mathrm{Ca}^{2+}\right]_{\mathrm{i}}$ was significantly increased with ATXII $(2.30$ $\pm 0.05)$ when compared to control $(0.96 \pm 0.08 ; \mathrm{n}=3 ; \mathrm{p}<0.01)$ and Ran fully reversed ATXII effect $(0.94 \pm 0.02 ; n=3$; $\mathrm{p}<0.01)$. Then, the effects of raised $\left[\mathrm{Ca}^{2+}\right]_{\mathrm{i}}$ by ATXII on Bmal1 rhythmicity was assessed using BAPTA-AM. Indeed, addition of BAPTA-AM prevented ATXII-induced Bmal1 period shortening (ATXII 17.09 $\pm 1.21 ;$ ATX+BAPTA-AM $29.91 \pm 0.54 ; \mathrm{n}=8 ; \mathrm{p}<0.05)$.

This study showed that in HL-1-6 cells only but not MEFs:1)ATXII treatment leads to a significant shortening of Bmal1 driven bioluminescence by almost 9 hours; 2)ATXII effect is fully reversed by ranolazine; 3)ATXII effects are mediated by raised $\left[\mathrm{Ca}^{2+}\right]_{\mathrm{i}}$ and hence was blocked by BAPTAAM. In conclusion, this study suggests a possible correlation between ATXII-induced atrial arrhythmias and Bmal1 gene and hence proposing a role for $\mathrm{Na}_{\mathrm{p}}$ activation channels in the disruption of atrial circadian rhythm.

\section{A HIGH THROUGHPUT SIRNA SCREENING IDENTIFIES RAC1 AS A POTENTIAL MODULATOR OF THE ENDOCYTOSIS OF THE TYROSINE KINASE RECEPTOR TIE2}

Marta Giralt-Pujol* ${ }^{*}$ Elizabeth Smythe. University of Sheffield

\subsection{6/heartjnl-2017-311726.149}

The Tie2 receptor is a cell surface tyrosine kinase receptor expressed almost exclusively in endothelial cells, where it is mainly implicated in angiogenesis. Indeed, Tie2 has been related to various pathologies with vascular defects such as pulmonary hypertension, diabetes retinopathy and tumour growth. Tie 2 activation and function is regulated in a complex manner by multiple factors that are still being investigated. For instance, after activation by the agonistic ligand Angiopoietin-1 (Ang1), Tie2 is internalised in cells by endocytic mechanisms that are yet to be fully characterised. As it has been shown that endocytosis can play a regulatory role in signalling, our hypothesis is that the endocytosis of Tie2 may also be important in the regulation of its activity and cellular output. Therefore, we decided to characterise the endocytic mechanisms involved in the internalisation of Tie2 to determine whether endocytosis can be a regulator of Tie2 signalling.

To facilitate the study of Tie 2 we created a HeLa cell line with inducible expression of a Tie $2^{\text {FLAG }}$ receptor that reflects expression levels of endogenous Tie2 in Human Umbilical Vein Endothelial Cells (HUVECs). HUVECs were isolated from umbilical cords anonymously donated in the Sheffield Hallamshire Hospital. To study the endocytosis of Tie2 receptor we developed a cell immunofluorescence-based internalisation assay to quantify the amount of internalised agonistic ligand Ang1 in a High Throughput Screening format (HTS).

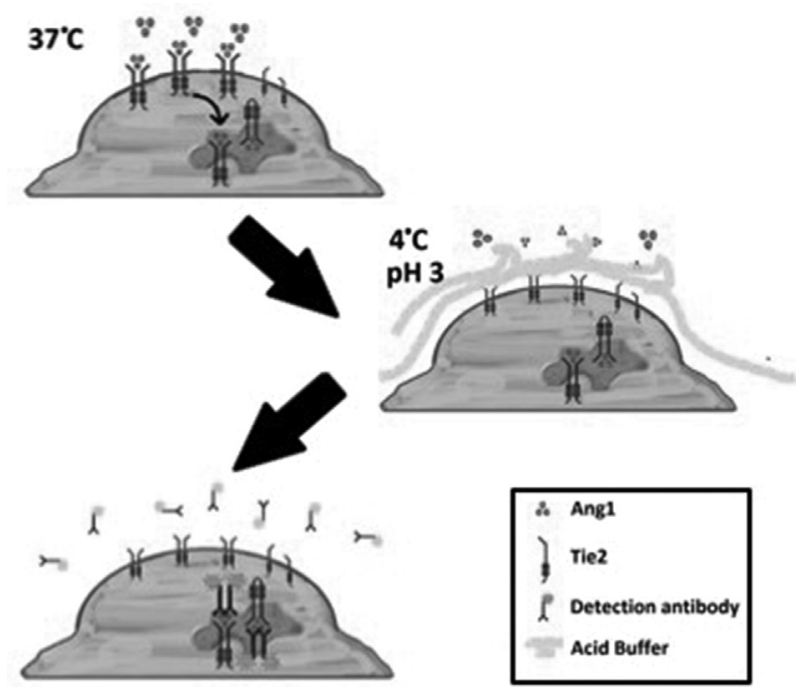

Abstract 150 Figure 1

Using the HTS internalisation assay we screened in HeLa/ Tie $2^{\text {FLAG }}$ cells a library of siRNAs targeting a selection of proteins involved in membrane trafficking. A selection of hits were selected for a secondary screen using different siRNAs and a pilot validation screen was performed using HUVECs. Interestingly, components of both clathrin-dependent and independent internalisation such as clathrin, AP2 subunits or caveolins were highlighted as potential regulators of the membrane trafficking of Tie2. Remarkably, the most robust hits along the different screens were two subunits of the actin related protein $2 / 3$ complex (ARP2/3) and the GTP binding proteins Dnm2 and Rac1. Along with Rac1, the Rac1-activated kinase Pak1 was also highlighted as a potential regulator of the internalisation of Tie2.

It was especially relevant to find Rac1 as a robust potential modulator of the internalisation of Tie2, as Rac1 is activated by the signalling cascade triggered by Tie 2 phosphorylation and it is implicated in physiological and cancer angiogenesis. This result highlights a potential cross-talk between the endocytosis and signalling of Tie2 that we believe needs to be further investigated. 